\title{
Identification of genomic indels and structural variations using split reads
}

\author{
Zhengdong D Zhang ${ }^{1 *}$, Jiang Du², Hugo Lam³ ${ }^{3}$ Alex Abyzov ${ }^{1}$, Alexander E Urban ${ }^{4}$, Michael Snyder ${ }^{5}$ and \\ Mark Gerstein ${ }^{1,2,3 *}$
}

\begin{abstract}
Background: Recent studies have demonstrated the genetic significance of insertions, deletions, and other more complex structural variants (SVs) in the human population. With the development of the next-generation sequencing technologies, high-throughput surveys of SVs on the whole-genome level have become possible. Here we present split-read identification, calibrated (SRiC), a sequence-based method for SV detection.

Results: We start by mapping each read to the reference genome in standard fashion using gapped alignment. Then to identify SVs, we score each of the many initial mappings with an assessment strategy designed to take into account both sequencing and alignment errors (e.g. scoring more highly events gapped in the center of a read). All current SV calling methods have multilevel biases in their identifications due to both experimental and computational limitations (e.g. calling more deletions than insertions). A key aspect of our approach is that we calibrate all our calls against synthetic data sets generated from simulations of high-throughput sequencing (with realistic error models). This allows us to calculate sensitivity and the positive predictive value under different parameter-value scenarios and for different classes of events (e.g. long deletions vs. short insertions). We run our calculations on representative data from the 1000 Genomes Project. Coupling the observed numbers of events on chromosome 1 with the calibrations gleaned from the simulations (for different length events) allows us to construct a relatively unbiased estimate for the total number of SVs in the human genome across a wide range of length scales. We estimate in particular that an individual genome contains $\sim 670,000$ indels/SVs.
\end{abstract}

Conclusions: Compared with the existing read-depth and read-pair approaches for SV identification, our method can pinpoint the exact breakpoints of SV events, reveal the actual sequence content of insertions, and cover the whole size spectrum for deletions. Moreover, with the advent of the third-generation sequencing technologies that produce longer reads, we expect our method to be even more useful.

Keywords: insertion, deletion, structure variation, split read, high-throughput sequencing

\section{Background}

One important goal in genomics is to determine the genetic differences among individuals and to understand their relationships to the phenotypic differences within a species, such as human beings. These variations consist of single nucleotide polymorphisms (SNPs) and structural variations (SVs) including short insertions/deletions (indels) and other more complex ones such as duplications and translocations. Because of the efficiency

\footnotetext{
* Correspondence: zhengdong.zhang@einstein.yu.edu; mark.gerstein@yale. edu

'Department of Genetics, Albert Einstein College of Medicine, Bronx, NY 10461, USA

Full list of author information is available at the end of the article
}

of genotyping methods and the central role they play in the genome-wide association studies, SNPs are currently the best catalogued and studied human genetic variations. Ubiquitous 1-bp indels, expansions of simple repeats and chromosomal anomalies have long been observed and acknowledged as the genetic bases for some human diseases [1,2]. Except for these old discoveries, however, indels and SVs have been much less studied due to their wide size range, the multitude in their types, and the lack of an efficient genotyping method. After several recent studies, however, their genetic significance starts to be appreciated: not only do they exist in large numbers in the human populations, they may
C Biomed Central

(C) 2011 Zhang et al; licensee BioMed Central Ltd. This is an Open Access article distributed under the terms of the Creative Commons Attribution License (http://creativecommons.org/licenses/by/2.0), which permits unrestricted use, distribution, and reproduction in any medium, provided the original work is properly cited. 
also have a more significant impact on phenotypic variation than SNPs [3-7].

The microarray technology, array $\mathrm{CGH}$, has been widely used to detect copy number variants (CNVs), a type of SV, with kilo-bases resolutions [5,8-11]. The advancement in high throughput sequencing technologies has enabled a new set of comparative approaches for CNV calling, such as the read-depth analysis [12-15], which computes the read coverage of different genomic regions, the read pair analysis, which focuses on cases where the distance between the two ends of a reads deviates more than expected when they are mapped back to the reference [4,16-18]. Accompanying the advancement of these experimental approaches, different computational methods for SV detection and their breakpoint refinement have also been developed [18-25].

Because indels/SVs come in various sizes, there is an additional aspect-the size coverage-to their detection. The aforementioned methods only partially address all the requirements of indel/SV detection to various degrees. For sequence insertions and deletions, indels/ SVs are conventionally defined as micro-SVs of 1-10 bp and large ones over $1 \mathrm{~kb}$, respectively. In the following text, wherever the context is clear we use SV as the encompassing term, subsuming small indels. Due to methodological limitations, SVs of middle lengths have only been minimally, if not at all, studied. Indeed, over the full spectrum of the SV size, only several small size spans are covered by current methods (Figure 1). Moreover, SV detection approaches described above (e.g. array/read-pair/read-depth based methods) cannot accurately locate the breakpoints of the SV events, nor can they reveal the actual sequence content of insertions. Such information can only be gained via the direct analysis of the read sequences, instead of based on the statistics of the mappings of such reads.

Here we report the split-read analysis, a sequencebased method that detects SVs through direct analysis of the mapping information of how high-throughput sequencing reads are aligned to the reference genome. Using alignment of read sequences to reference genomes with gaps, the method allows the precise identification of SVs covered by such reads. Building our method directly upon BLAT, a well-established sequence alignment program, we take advantage of the speed and the sensitivity of this popular sequence-togenome alignment tool. However, more importantly, by considering both the sequencing and mapping errors in our assessment strategy to score each initial SV call, our method also takes into account the sequencing error model (especially for next-generation sequencing technologies, which were not generally available a few years ago), and distinguishes the different confidence levels in detecting different SVs based on the characteristics of supporting reads. Compared with the read-depth and the read-pair analyses, our sequence-based method can not only pinpoint the breakpoints of SV events, but also reveal the actual sequence content of insertions. The split-read analysis has another advantage-it can cover the whole size spectrum for deletions (Figure 1). We expect our method to be more useful in the future as the sequence reads become longer.

Due to both experimental and computational limitations, there are biases on multiple levels in the call sets generated by all current SV identification methods. In addition to their significantly more restricted size range of identifiable insertions than that of deletions, all current SV identification methods are sensitive to SVs of different length (Figure 1), and as a result studies using them have reported different numbers of SVs. One study using the read-pair method reported 241 SVs over $8 \mathrm{~kb}$ in a sampled genome [7], while another using the same approach but with a different molecular construct reported 422 and 753 SVs over $3 \mathrm{~kb}$ in two tested genomes [4]. In a study of whole-genome sequencing and assembly, 835,926 indels were identified in a diploid human genome [26]. Currently it is not known how many SVs, small or large, are in an individual human genome. Using empirical error models estimated from sequencing experiments to simulate high-throughput sequencing reads, we could not only parameterize our split-read method, but also, more importantly, quantify both false positive and false negative rates. Knowing these error rates enables us to estimate the total number of SVs of a given length in a human genome.

\section{Results}

We have developed the split-read identification, calibrated (SRiC), a sequence-based method for detecting structural variants (SVs). It maps reads to the reference genome with gapped alignment and scores these mappings with consideration for sequencing and alignment errors. SRiC pinpoints exact SV breakpoints, reveals the sequence content of insertions, and covers the whole size spectrum for deletions. Simulation is used to calibrate SRiC, allowing unbiased estimation of the sensitivity and proportion of SVs across different length-scales.

\section{Analysis of the simulated sequence data}

For sequencing simulations, instead of using the whole human genome, we use the diploid human chromosome 22 (NCBI36 assembly), which counts for $1 \%$ of the human genome but has a repeat content and a gene density both representative of the whole genome, to save computational processing time. To keep the local sequence environment of indels as found in a genome, we use indels identified in Venter's genome [26] in our sequencing simulation (Additional file 1). 


\section{Deletions}

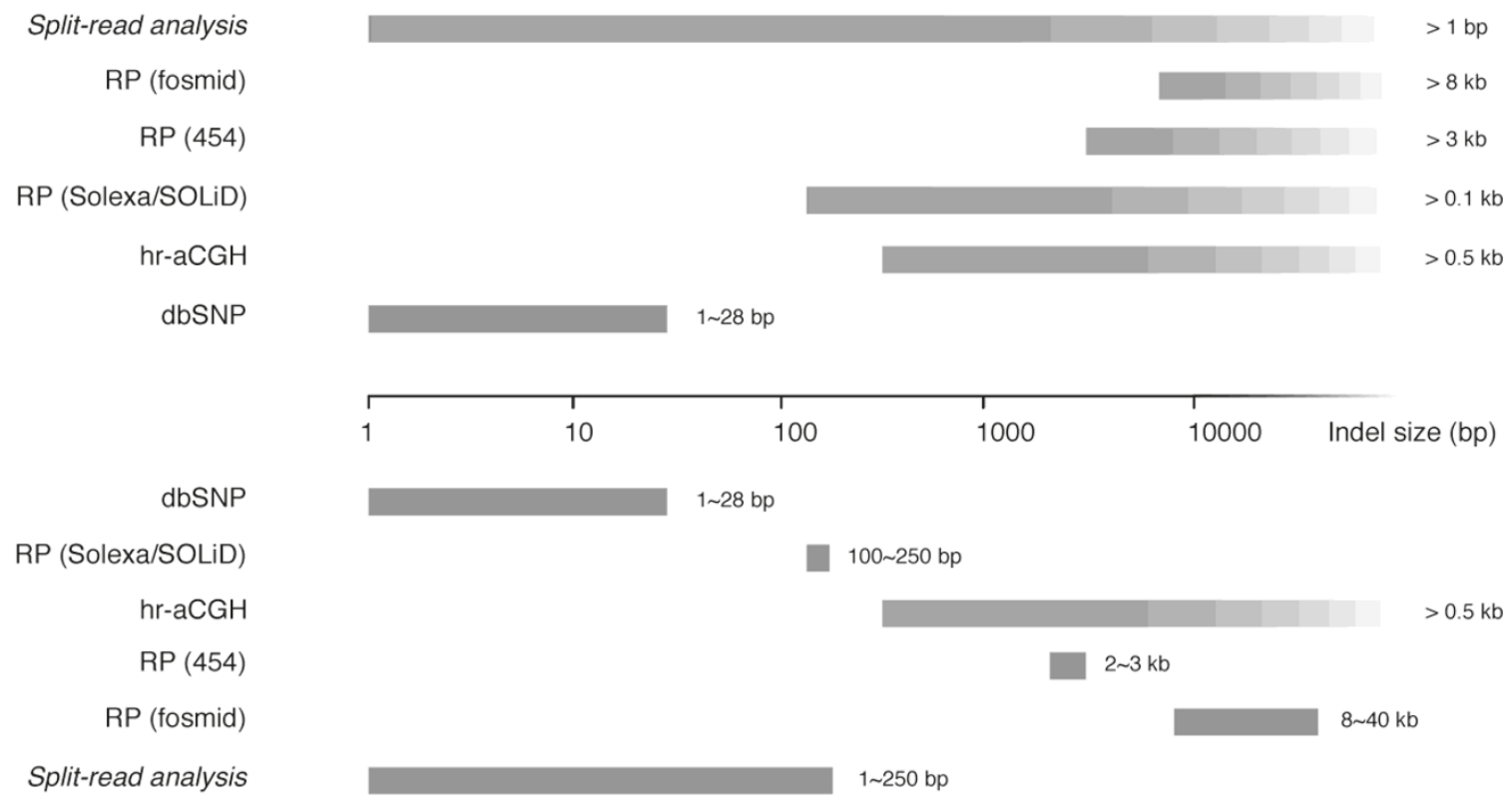

\section{Insertions}

Figure 1 The size spectrum of SVs identifiable to different methods. No method can identify SVs of all different sizes. The black bars indicate the size ranges of discoverable SVs by different methods, which include the dbSNP database, the high-resolution array CGH (hr-aCGH), the read-pair (RP) method with fosmid, 454, and Solexa sequencing, and the split-read analysis. The range of detectable indels by RP depends on three values: the mean and the standard deviation between the distances of mapped read pairs and the multiple coefficient of s.d. for significance. These triple values are (40 kb, 2.8 kb, 3), (1 kb, 0.8 kb, 3), (250 bp, 25 bp, 6) for fosmid, 454, and Solexa sequencing, respectively.

\section{Determining thresholds used in the analysis}

Three thresholds are used in our split-read analysis: $t_{\mathrm{r}}$, the threshold on the ratio of the score of the best alignment to that of the second best as a measure of the uniqueness of the read, $t_{\mathrm{n}}$, the threshold on the number of supportive reads for 1-bp SVs, and $t_{\mathrm{c}}$, the threshold on the maximum centeredness (the maximum ratio of the smaller length to the bigger one of two flanking alignments of a read, Additional file 1, Figure S1) for large SVs.

To determine the score ratio threshold $t_{\mathrm{r}}$ for the alignment preprocessing, we simulate $\sim 5 \times$ sequence coverage that gives $\sim 0.6$ million 454 single-end 400 -bp reads and then identify SVs using different values for the score ratio threshold $t_{\mathrm{r}}(=1.0,1.25,1.5,1.75$, and 2.0$)$ while keeping the other two parameters fixed $\left(t_{\mathrm{n}}=5, t_{\mathrm{c}}=0.1\right)$. The percentage of true positives, false negatives, and false positives of deletions and insertions identified at different $t_{\mathrm{r}}$ values are plotted in Figure 2A-B. There is a small decrease in the number of identified SVs when $t_{\mathrm{r}}$ is increased from 1.0 to 1.25 . The further increases in $t_{\mathrm{r}}$ from 1.25 to 2.0 only cause negligible changes to the SV identification results. Over all, the SR method is not very sensitive to $t_{\mathrm{r}}$ when it is in the range of 1.0 and 2.0.
This insensitivity is a result of unique mapping to the reference genome of most 454 reads, which are much longer than those produced by other next-generation sequencing technologies.

Two thresholds, $t_{\mathrm{n}}$ and $t_{\mathrm{c}}$, are used for the initial SV calls (Inequalities 1 and 2). We vary the value of one of these two thresholds while fix the other to determine how they affect the accuracy and the sensitivity of the split-read method. Using the simulated sequence set with the $\sim 5 \times$ coverage, we make SV calls with $t_{\mathrm{n}}=1,2$, ..., 9 while $t_{\mathrm{c}}=0.1$ and $t_{\mathrm{c}}=0.1,0.2, \ldots, 0.9$ while $t_{\mathrm{n}}=5$, count the true positive and the false positive calls, and calculate the percentage of true positives, false negatives, and false positives at each threshold combination. The results of this performance analysis as depicted in Figure 2C-F make it clear the effects that theses two thresholds have on the SV identification show a dichotomous dependency on the SV length. While $t_{\mathrm{n}}$ affects the identification of short SVs, $t_{\mathrm{c}}$ biases that of longer ones. In practice, we use the sequencing depth for $t_{\mathrm{n}}$ (with a lower bound $n_{\text {min }}=2$ ) and set $t_{\mathrm{c}}$ to 0.1 . It is also clear that the method has different sensitivities in the size range of indels that it can detect: it is less sensitive to 1bp indels because 454 sequencing is prone to over- or 


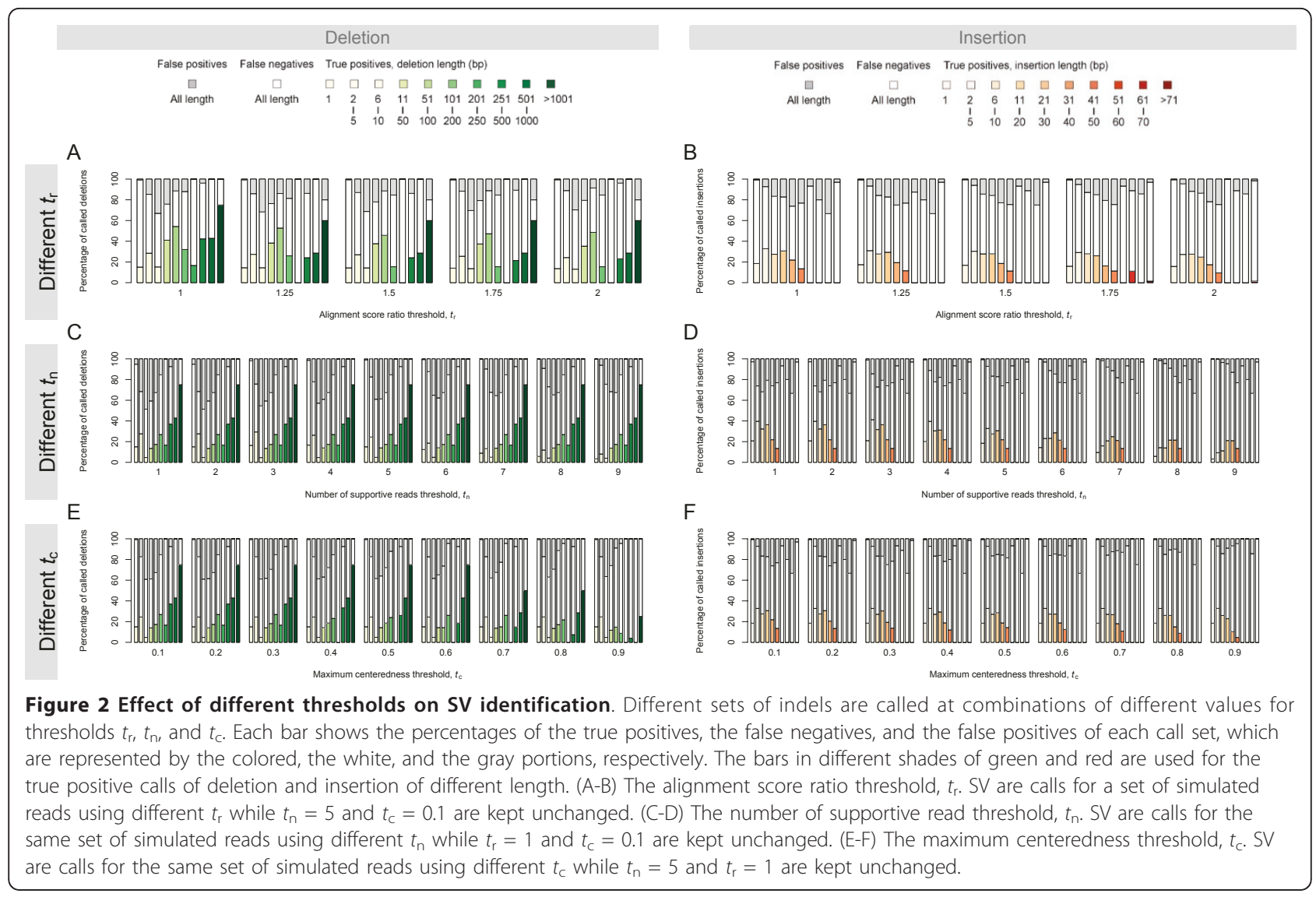

under-call bases in homopolymers and thus more a stringent threshold is needed to lower the number of 1bp false positives.

\section{Assessing how the read length affects the performance}

We first assess how the read length affects the SV identification by simulating single-end reads of 50, 100, 200, 400 , and $800 \mathrm{bp}$ long. For each read length, we generate sequences with $\sim 5 \times$ coverage and analyze five sequence sets with the same set of method parameters $\left(t_{\mathrm{r}}=1, t_{\mathrm{n}}\right.$ $\left.=5, t_{\mathrm{c}}=0.1\right)$. We compare the true SVs and the ones that we identified using the split-read analysis. The numbers of true and false positives of deletions and insertions identified using reads of different lengths are plotted in Figure 3A-B.

The general trend, which is expected and depicted in the figure, is that the SV identification is improved with longer reads. With 50-bp reads, the SV identification is the worst with low sensitivity for both short and long deletions. Because the length of discoverable insertions is capped by the read length, it is not surprising that at this read length none of the insertions of 20-bp and longer are found. When the read length is increased to $200 \mathrm{bp}$ and longer, the sensitivity and the positive predictive value almost double for longer SVs. For deletions, 200-, 400-, and 800-bp reads seems to give comparable performance, and longer reads only bring marginal improvements to the results. The choice of read length for insertions identification is, however, a rather open-end question, as longer reads will always enable better identification of longer insertions.

\section{Assessing the effects of sequence coverage on SV calls}

We first simulate $\sim 20 \times$ sequence coverage that gives $\sim 2.5$ million 454 single-end 400-bp reads. To assess how the sequencing depth affects the SV calls by the splitread analysis, we also simulate $\sim 1 \times, 5 \times, 10 \times$, and $15 \times$ sequencing coverage by down-sampling the $20 \times$ sequence set with appropriate numbers of reads (Table $1)$. We then identify SVs using default parameters $\left(t_{\mathrm{r}}=\right.$ $1.0, t_{\mathrm{n}}=$ coverage, $t_{\mathrm{c}}=0.1$ ). The numbers of true and false positives of deletions and insertions identified at different sequencing coverage are plotted in Figure 3CD. The general trend is that SV identification is improved with higher coverage but with diminishing returns. Comparing to the low coverage at $1 \times$, there is a marked improvement to SV identification at higher coverage.

To assess how sequencing coverage affects the sensitivity of our method, we determine the maximum sensitivity achievable in each simulated sequence set. The number of 'seeable' true SVs is affected by several 


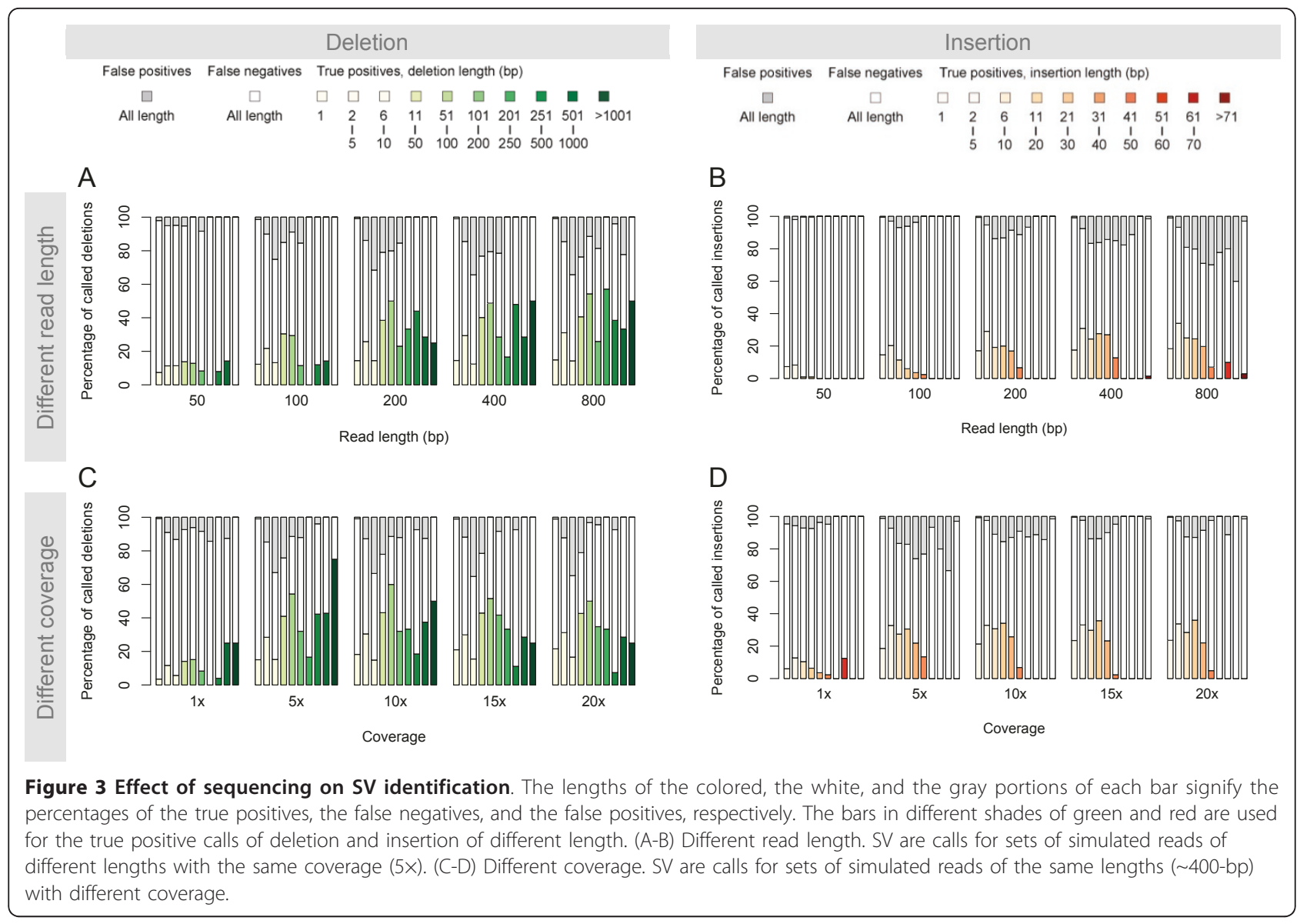

factors, including the sequencing depth, the read mapping quality/uniqueness, and the minimum number of supportive reads required for an SV call. After the initial alignment processing to remove the mapping ambiguity, we count the number of supportive reads for the true SVs of different lengths and plot the number of true

Table 1 Number of sequences in simulated and downsampled datasets

\begin{tabular}{llll}
\hline Sequence type & Coverage & $\begin{array}{l}\text { Number of } \\
\text { sequences }\end{array}$ & $\begin{array}{l}\text { Number of base } \\
\text { pairs }\end{array}$ \\
\hline $\begin{array}{l}\text { Generated } \\
\text { sequences }\end{array}$ & $20 \times$ & $2,477,629$ & $994,491,814$ \\
$\begin{array}{l}\text { Mapped } \\
\text { sequences }\end{array}$ & $20 \times$ & $2,476,347$ & $993,977,159$ \\
Used sequences & $20 \times$ & $2,476,088$ & $993,873,276$ \\
& $15 \times$ & $1,857,784$ & $745,693,367$ \\
& $10 \times$ & $1,236,929$ & $496,489,303$ \\
& $5 \times$ & 619,052 & $248,478,809$ \\
& $1 \times$ & 123,633 & $49,625,857$ \\
\hline
\end{tabular}

Notes:

1. We use $49,691,432$ bp as the size of human chromosome 22 for calculating the sequence coverage.

2. For $1 \sim 15 \times$ target sequence coverage, we sample from the used sequences in the $20 x$-coverage dataset.
SVs with one and two or more supportive reads at different sequencing depth (Figure 4).

The sequencing depth has the most significant effect on short SVs. At $1 \times$ coverage, 1,000 of true 1-bp deletions and insertions are supported by at least one read. When the coverage is increased to $2 x$, these numbers almost are doubled. As the coverage increases, the percentage of supported true SVs also increases but with a diminishing pace. $80 \sim 90 \%$ true SVs are supported by at least one read at $5 \times$ to $20 \times$ coverage. One supportive read is the absolutely minimum requirement for an SV call. To reduce the false positives, we require at least two supportive reads for every SV call. This global threshold has a much more significant effect on the low-coverage sequence set than on the high-coverage one: while the percentage of true deletions with two or more supportive reads is about the same as that of true deletions with one supportive read at $1 \times$ coverage, there are very few true SVs with only one supportive read at $10 \times$ or higher coverage.

\section{Performance assessment}

Several different approaches have been used to extensively evaluate the performance of our SRiC method 


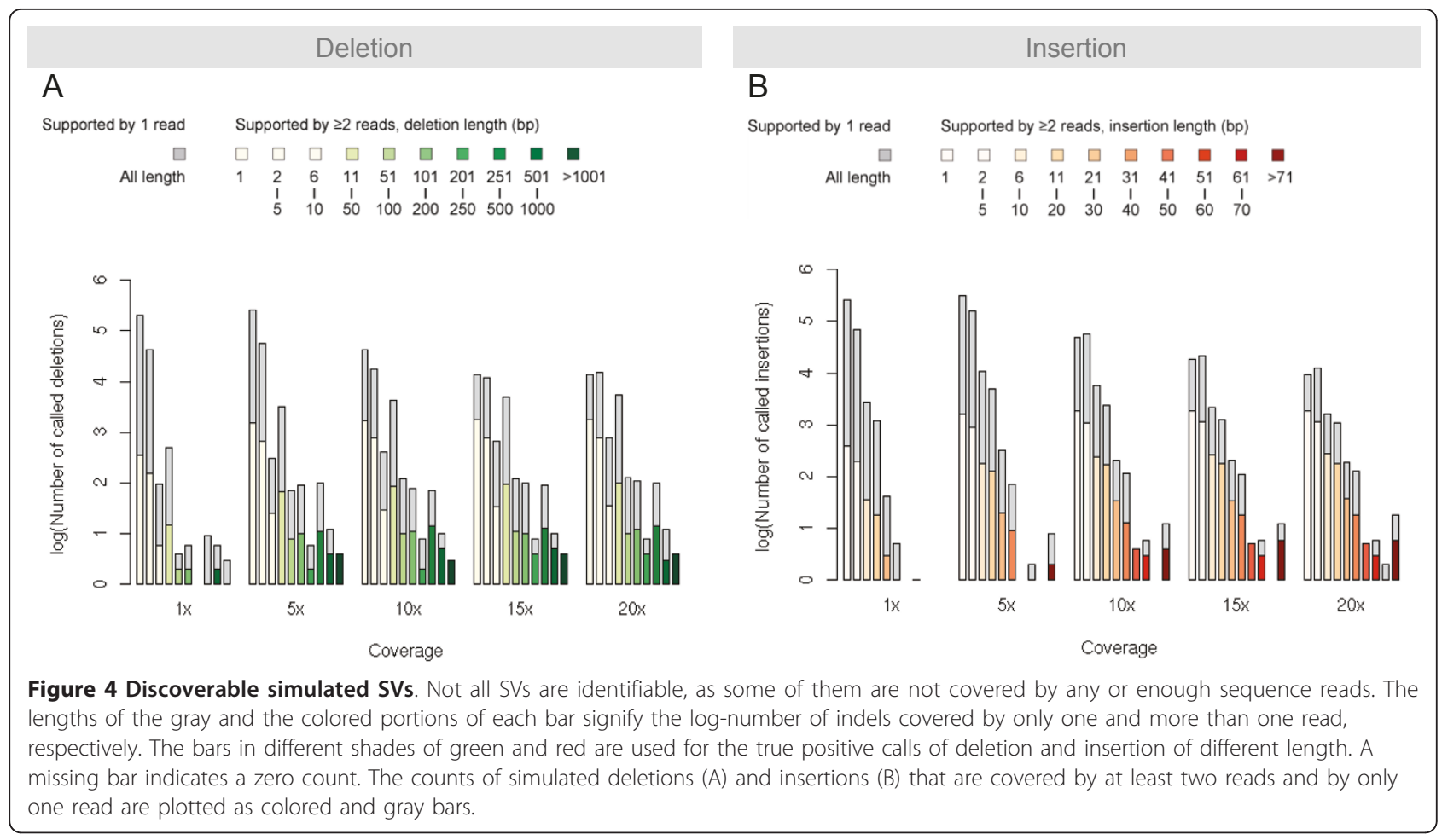

(Additional file 1). First, we compare SRiC with Pindel, the only published method that can detect SV breakpoints on the nucleotide level. The comparison between the numbers of SVs that these two methods can find in simulated datasets with the same SV placements shows SRiC has a significantly higher sensitivity than Pindel at every length simulated, whether it is of deletions or insertions (Additional file 1, Tables S1 and S2). Second, we apply our split-read analysis to 454 genomic reads generated for two individuals (CEU NA12878 sequenced to $0.5 \times$ and YRI NA19240 to $5 \times$ ) and calculate the positive predictive values at different thresholds on the number of supportive reads after validating deletion calls using two experimental methods, respectivelyarray capture followed by sequencing and trio-array comparative genomic hybridization $(\mathrm{CGH})$. The experimental result shows for the type of SVs under consideration SRiC can achieve 70-80\% call accuracy (Tables 2 and 3).

\section{Analysis of the 1000 Genomes Project data}

A major sequencing project, the 1000 Genomes Project, has been launched to resequence the genomes of at least a thousand people from around the world using the new sequencing technologies to produce the most detailed map of human genetic variation for disease studies. As a proof of concept, we apply our split-read analysis to a set of 454 sequence reads generated by the 1000 Genomes Project for one individual.
The genome of an individual (NA19240) from the Yoruba in Ibadan, Nigeria has been sequenced using the 454 single-end method to $\sim 5 \times$ sequence coverage. The sequencing generated $\sim 49$ million sequence reads, of $\sim 17.6 \times 10^{9} \mathrm{bp}$ in total. Mapping of the sequence reads (with a median length of $\sim 400 \mathrm{bp}$ ) took $\sim 136$ hours of the wall time with exclusive access to 50 Dell PowerEdge 1955 nodes (each containing 2 dual core $3.0 \mathrm{Ghz}$ Xeon 64 bit EM64T Intel CPUs model 5160 and 16 GB RAM) of a Linux cluster. Applying our SR method, we identify 13,426 deletions ranging from $1 \mathrm{bp}$ to $\sim 700 \mathrm{~kb}$ and 11,539 insertions ranging from $1 \mathrm{bp}$ to $200 \mathrm{bp}$ on the chromosome 1 . Compared with 494 validated insertions in chromosome 1 from dbSNP (v129), 301 in both sets are at exactly the same genomic locations, which indicates a sensitivity of $\sim 60 \%$ for validated insertions in

Table 2 Array capture validation of SR called deletions. ${ }^{1}$ 2

\begin{tabular}{lllllll}
\hline $\boldsymbol{t}_{\mathbf{n}}{ }^{\mathbf{3}}$ & $\mathbf{2}$ & $\mathbf{3}$ & $\mathbf{4}$ & $\mathbf{5}$ & $\mathbf{6}$ & $\mathbf{7}$ \\
\hline True positive & 30 & 20 & 15 & 13 & 9 & 7 \\
Positive & 115 & 33 & 22 & 18 & 12 & 10 \\
Positive predictive value $^{4}$ & 0.26 & 0.60 & 0.68 & 0.72 & 0.75 & 0.70
\end{tabular}

Notes:

1. $0.5 \times 454$ reads for a CEU individual (NA12878) from the 1000 Genomes Project are used to make the SR deletion calls.

2. Only deletions longer than $500 \mathrm{bp}$ are selected for array capture validation. 3. $t_{\mathrm{n}}$, is threshold on the number of supportive reads.

4. Positive predictive value $=$ True positive/Positive. 
Table 3 Trio-array CGH validation of result. ${ }^{1,2}$

\begin{tabular}{lllllll}
\hline $\boldsymbol{t}_{\mathrm{n}}{ }^{\mathbf{3}}$ & $\mathbf{2}$ & $\mathbf{3}$ & $\mathbf{4}$ & $\mathbf{5}$ & $\mathbf{6}$ & $\mathbf{7}$ \\
\hline True positive & 48 & 23 & 17 & 12 & 9 & 7 \\
Positive & 76 & 27 & 20 & 15 & 11 & 9 \\
Positive predictive value $^{4}$ & 0.63 & 0.85 & 0.85 & 0.80 & 0.82 & 0.78 \\
\hline
\end{tabular}

Notes:

1. Only deletions longer than $50 \mathrm{bp}$ called for an YRI individual (NA19240) from the 1000 Genomes Project are selected (randomly) for the trio-array CGH validation. Due to the data usage restriction, only validation results for deletions in chromosome 1 are used.

2. Not every validation test yields definite result. Inconclusive results are excluded from this table.

3. $t_{n}$, threshold on the number of supportive reads.

4. Positive predictive value $=$ True positive/Positive.

dbSNP. This defines a lower bound on sensitivity as different genomic DNA sources are involved. The simulation used to compare the numbers of insertion and deletion calls (see above) enables us to determine the positive predictive values and the sensitivities of our SR method for indels identified in a sequence set at $5 \times$ coverage and subsequently estimate using equation (4) the total numbers of deletions and insertions of lengths in continuous ranges separately on chromosome 1 (Table $4)$. We estimate there are 53,431 SVs in chromosome 1 and extrapolate to $665,684 \mathrm{SVs}$ in the whole genome of this individual.

\section{Discussion}

\section{Mapping reads to the reference genome}

The size of the deletions covered by the split-reads can range up to tens of thousands of bases, and this makes BLAT well suited for mapping such reads back to the genome, since it not only allows small gaps and mismatches within the alignment like many other alignment tools, but also takes into account large gaps due to its initial purpose to handle introns in RNA/DNA alignments [27]. In short, unlike the alignment results from tools such as BLAST which will generate two distinct partial alignments for a split-read covering a large deletion event, the alignment results of BLAT can directly reveal the deletion event and its up- and down-stream alignments at the same time. Recently a new algorithm, Burrows-Wheeler Aligner's Smith-Waterman Alignment (BWA-SW), has been designed and implemented to align with gaps long reads such as 454 reads ( $200 \mathrm{bp}$ or longer) to the reference genome with higher accuracy and a faster speed than BLAT [28]. However, BLAT should be used to align 454 paired-end reads, because currently the average 454 read length is less than 400 bp and thus, the majority of sequences on both ends will be shorter than $200 \mathrm{bp}$.

For the non-split reads, however, using BLAT would be unnecessarily time-consuming, because their alignment results would usually only contain (if any) a small number of mismatches. Bowtie, a recently developed alignment tool, incorporates the Burrows-Wheeler transform technique to index and search the genome in a fast and memory-efficient manner, and is an immediate candidate for processing such reads [29].

The two-tiered alignment cascade is used to expedite the step of aligning reads to the reference genome. The first assortment step effectively fractions the sequence reads into two subsets: ones that can be uniquely mapped and ones that cannot. By limiting the gapped alignment of the reads in the former subset to their associated chromosomes, the tiered mapping approach removes the unnecessary mapping attempts and thus speeds up the alignment step. The speed gain is clearly related to the size ratio of the two read subsets: the more uniquely mappable reads, the bigger the speed gain. Because it is assessed by their 35-bp end tags, the genomic uniqueness of the reads is limited to the unique mappability of the 35 -mers to the human genome. It has been estimated that $79.6 \%$ of the genome is uniquely mappable using 30 bp sequence tags. Since the human genome consists of 24 chromosomes, it is natural to use them as the bins for end tag assortment. It is, however, conceivable to fraction the human genome into large (e.g., $100 \mathrm{Mb}$ ) fragments with small (e.g., $1 \mathrm{~kb}$ ) overlaps and use them as the assortment bins to further restrict the search space of the

Table 4 Corrected counts of SVs in the chromosome 1 and the whole genome of a Yoruba individual ${ }^{1}$

\begin{tabular}{|c|c|c|c|c|c|c|c|c|c|c|}
\hline \multirow{2}{*}{$\frac{\text { SV type }}{\text { Deletion }}$} & \multicolumn{10}{|c|}{ SV size range (bp) } \\
\hline & $1-5$ & $6-10$ & $11-50$ & $51-100$ & $101-200$ & $201-250$ & $251-500$ & $501-1000$ & $>1000$ & Total \\
\hline Chromosome 1 & 20,229 & 3,018 & 1,619 & 183 & 224 & 156 & 419 & 37 & 101 & 25,986 \\
\hline Whole genome ${ }^{2}$ & 252,028 & 37,600 & 20,171 & 2,280 & 2,791 & 1,944 & 5,220 & 461 & 1,258 & 323,753 \\
\hline Insertion & $1-5$ & $6-10$ & $11-20$ & $21-30$ & $>30$ & Total & & & & \\
\hline Chromosome 1 & 22,187 & 3,743 & 1,074 & 228 & 213 & 27,445 & & & & \\
\hline Whole genome & 276,422 & 46,633 & 13,381 & 2,841 & 2,654 & 341,931 & & & & \\
\hline
\end{tabular}

Notes:

1. The true number of large SVs will be underestimated. However, this will have only a marginal effect the magnitude of the estimation of the total number of SVs in a large chromosome or in the whole genome.

2. The true number of SVs in the whole genome is estimated by extrapolation of the corrected number of SVs in chromosome 1 by the fold increase in size from chromosome 1 to the whole genome. 
subsequent BLAT genomic mapping of the reads whose end tags are uniquely mapped.

\section{Parameterization of the split-read analysis}

Five parameters are intrinsic to our split-read analysis alone: the alignment score ratio threshold $t_{\mathrm{r}}$, the threshold on the number of supportive reads for 1-bp SVs $t_{\mathrm{n}}$, the threshold on the maximum centeredness for large SVs $t_{\mathrm{c}}$, the minimum number of supportive reads for every SV identification $n_{\text {min }}$, and the exponential decay parameter $\lambda$. For sequence reads that are mapped to multiple genomic locations, we use $t_{\mathrm{r}}$ to control on what level of distinctiveness such reads can be used for the SV identification. A higher value of $t_{\mathrm{r}}$ lowers the overall mapping ambiguity and thus reduces the number of false positives. This will, however, disqualify more correct alignments and in turn increase the number of false negatives. Small and large false SV calls have different origins: the former result from sequencing errors that under- or over-call bases while the later are mostly generated by misalignments. To count for such distinct error origins, two different threshold functions, separately parameterized with $t_{\mathrm{n}}$ and $t_{\mathrm{c}}$ using the same exponential base function, are used to make SV calls. $\lambda$ controls how fast the threshold changes between 1-bp and large SVs and it is set to 1 in all of our split-read analyses. We require that there should be at least two supportive reads for every SV identified regardless of its length. This global threshold $\left(n_{\min }=2\right)$ dramatically reduces the false positive SV calls.

\section{Conclusions}

Directly building our method upon BLAT, we take advantage of the speed and the sensitivity of this popular sequence-to-genome alignment tool. However, more importantly, we designed an assessment strategy to score each initial indel/SV call that takes into account both the sequencing and mapping errors. Compared with the existing read-depth and read-pair analyses, our sequence-based method can pinpoint the exact breakpoints of indel/SV events, reveal the actual sequence content of insertions, and cover the whole size spectrum for deletions. We thoroughly benchmarked and validated our SRiC method against the best available methods for detecting structural variants at relevant resolutions by using several different approaches to extensively evaluate the performance of our method. We illustrate the characteristics of our split-read method by applying it to both synthetic and experimental data sets. With the advent of the third-generation sequencing technologies that produce longer reads, we believe the split-read approach presented here can make a significant contribution to the study of indels/SVs.

\section{Methods}

Sequence data are analyzed in a stepwise fashion, as depicted in Figure 5. Below we describe our split-read analysis in detail.

\section{Data input}

The data input for the split-read analysis are genomic read sequences. For sufficient alignability, these reads should have a length of hundreds of bases and currently can be generated by the Sanger sequencing or, to a much higher throughput, the 454 sequencing. However, we expect reads from other sequencing platform (e.g., paired Solexa reads with overlap) may also be used after preprocessing. The current system implementation only supports the widely used FASTA sequence format.

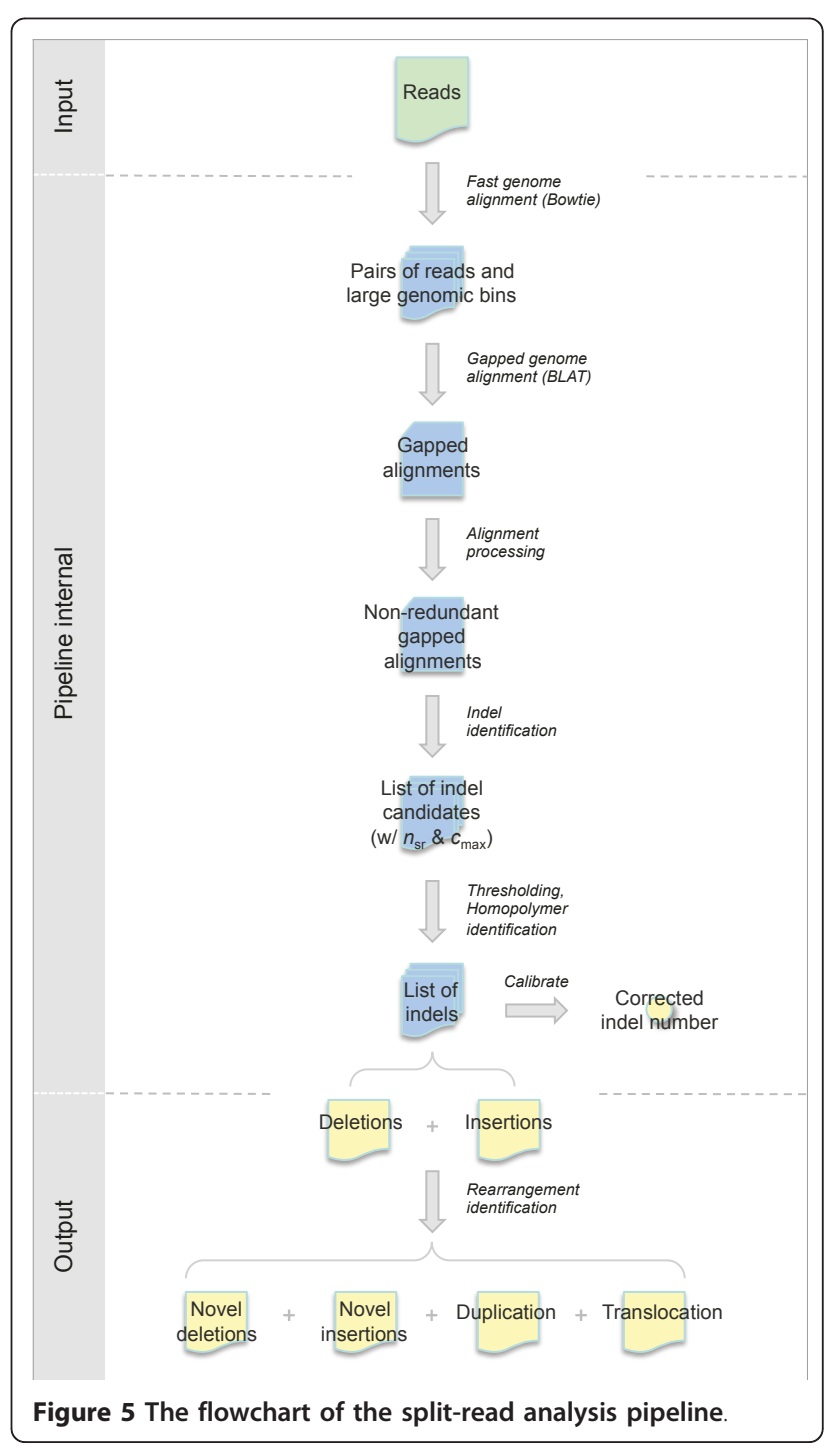




\section{Tiered sequence alignment}

The sequence reads are first processed to remove any terminal ambiguous bases (Ns) and then mapped to the human reference genome (NCBI Build 36.1, UCSC hg18) using BLAT with parameters tuned for short sequences with maximum sensitivity (-stepSize $=5$, -tileSize $=11$, -repMatch $=10^{6}$, and -fine). Certain parts of the reference genome (such as low complexity regions and simple sequence repeats) can be masked out by replacing the sequences with Ns to disallow indel identification in these regions. When the set of reads is large, the aforementioned direct approach to sequence mapping will be very time-consuming. To enhance the speed of the alignment step, we use a tiered approach instead by dividing our alignment process into two steps: a fast initial assortment of the reads followed by a complete gapped alignment.

Briefly, we first take 35-mer tags at each end of a read, map them to the whole reference genome using Bowtie, a rapid alignment tool for short reads, look for those end tags that can be mapped uniquely to the genome, and assort the corresponding reads by their associated chromosomes. Using BLAT to obtain the gapped alignments, we then align the assorted reads only to their targeted chromosomes and the remaining reads whose ends cannot be uniquely mapped to the whole genome. Thanks to the modularity of the implementation, Bowtie and BLAT used here can be replaced by other alignment tools, such as MAQ and BLAST, with minor modifications.

For all uniquely mappable reads, this tiered mapping approach can speed up the alignment to the human genome by 24 times on average. The whole process is parallelized, and for a total of $\sim 3$ million reads ( $\sim 60 \mathrm{~GB}$ in size) it takes less than an hour to finish the assortment step with 80 CPUs of a computer cluster. On average, $\sim 70 \%$ of the single-end reads of a sequenced individual could be assorted by the aforementioned algorithm. As a result, we anticipate an overall enhancement of the alignment speed by 3 folds.

\section{Alignment preprocessing}

If a read is mapped to the genome uniquely, we keep its alignment without additional requirements. Otherwise, its alignments are scored and the alignment ratios calculated. The alignments are then sorted on their scores, ratios, and the number of alignment blocks. We only keep the top alignment when its score is at least $t_{\mathrm{r}}$ times (to be determined by simulation) as big as that of the second best on the sorted list. Moreover, DNA amplification as part of the library preparation procedure increases the likelihood that a DNA fragment is sequenced multiple times. Redundant sequence reads (the same chromosome, the same strand, and the same start position) generated from the same DNA fragments are removed to prevent the inflation of the count of reads that are supportive of SVs.

For paired-end sequence reads, they are processed to release the end sequences with the pairing information preserved for later use after the linker sequence is identified and removed. The end sequences are then mapped and processed like the single-end reads as described above. Because of restriction on how two ends are mapped relatively to each other on the genome, the pairing information increases the accuracy of their genomic placement. To avoid excessive assumptions on the distribution of the insert length, we make the minimum requirement that two ends of a read should be mapped to the same strand of the same chromosome. Only read ends that make unique concordant pairs are used in the downstream analyses.

\section{Insertion/deletion and rearrangement identification}

After sequence alignment and placing reads at their most likely locations in the reference genome, the splitread analysis searches these locations for insertions and deletions in the sample genome by identifying reads that encompass SV break points (Figure 6). To find deletions in the sample genome, we search for reads that when aligned to the reference genome split on the same strand of a chromosome. Even though a deletion of an arbitrary size can be detected as long as it is covered by one or more reads, the size of insertions that can be directly detected in full is limited by the read length. To find small insertions that are fully included in the reads, we search for reads whose terminal sequences can be aligned next to each other on the reference genome. For large insertions, we look for their boundaries, which are found in reads that, except one of their ends, can be aligned to the reference genome continuously in one block.

For each identified SV, we count the number of reads that 'support' it, $n_{\mathrm{sr}}$, and measure its centeredness in each supportive read, $c_{i}\left(i=1, \ldots, n_{\mathrm{sr}}\right)$, the ratio of the smaller length of its two flanking alignments to the bigger one. It is easy to see that $0<c_{i} \leq 1$ and if there are multiple supportive reads for an SV it is the maximum centeredness that matters the most (because the evidence best supportive of presence is the most informative). Thus, each SV identification is associated with two scoring quantities: the number of supportive reads, $n_{\mathrm{sr}}$, and the maximum centeredness, $c_{\max }$ (Additional file 1 , Figure S1).

Considering the lists of deletions and (small) insertions together in conjunction with each other, we resolve their final SV identities as novel deletions, novel insertions, duplications, and translocations. To do this, we first extract from reads the sequences of insertion 


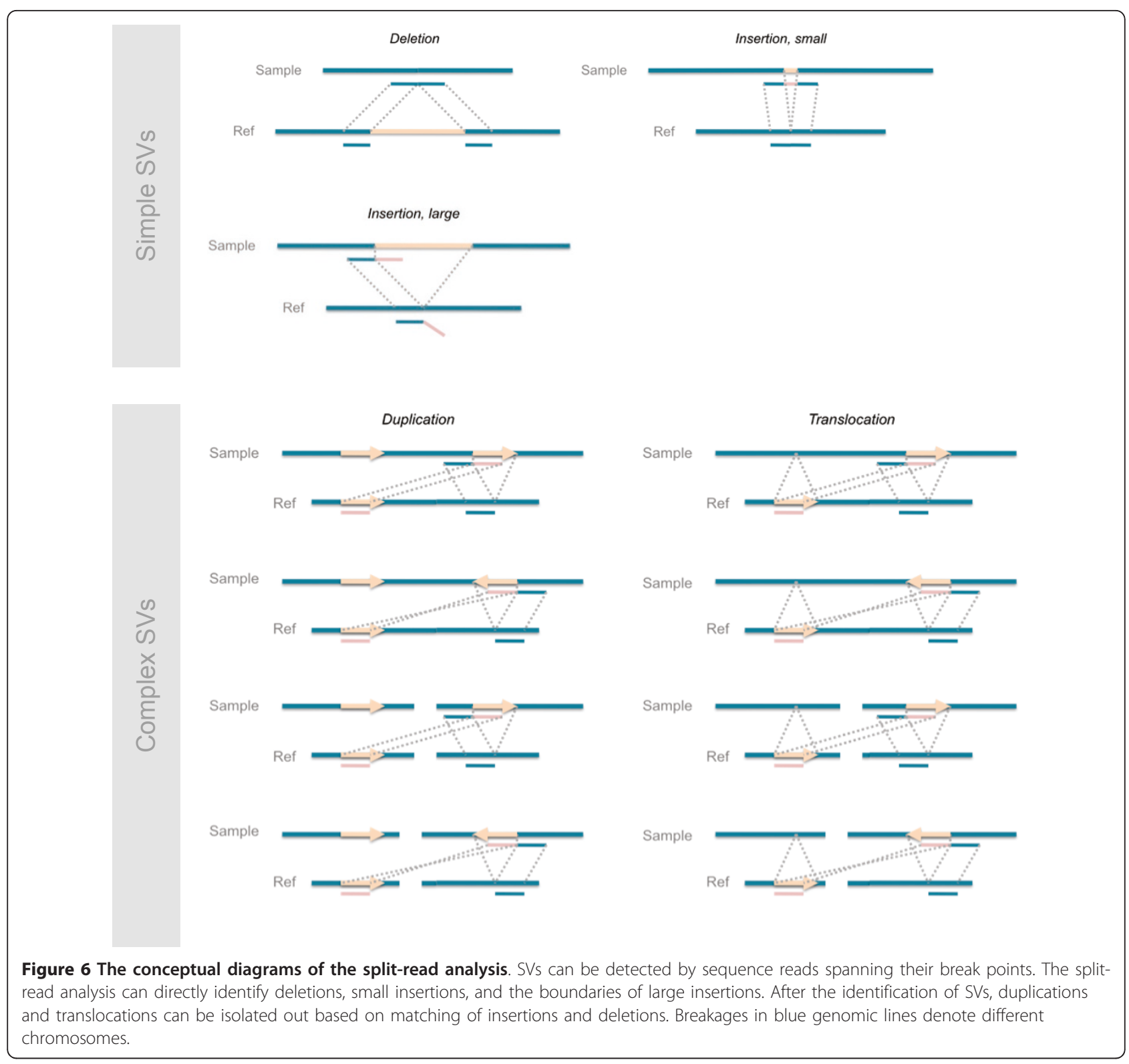

that are at least $20 \mathrm{bp}$ long and then align them to the reference genome using BLAT. An insertion is classified as 'novel,' if it cannot be aligned perfectly without gaps. Otherwise, it is a duplication and potentially a translocation. To be the latter, at least one location of the perfect alignments to the reference genome needs to be precisely covered by a read with deletion. The novel deletions are the whole set of deletions excluding those 'used' by translocations.

\section{SV call set obtention through SV call filtering and sequencing error identification}

Sequencing errors or spurious sequence alignments can both lead to SVs calls by the split-read analysis. The majority of such false positives can be removed by imposing a simple global threshold that requires every SV to be found in at least two nonredundant reads. We further refine the call list, and since the false positives of the short and the long SVs arise from distinct sequencing and alignment errors, respectively, we treat the short and the long SV calls differently.

Based on the SV length, false SV calls have different origins: small sequencing errors, large misalignments, and a mixture in between. Sequencing errors that under-or over-call bases manifest as deletions and insertions in the sequence reads when they are aligned to the reference genome. False SVs of this origin have the characteristics that they are very short, mainly 1-bp 
SVs, and also occur largely in a random fashion. In contrast, false large SV calls are mostly generated by misalignments in which the SVs are located very closely to one end of the reads. False SV calls with lengths in the narrow middle range are thought to be a mixture of errors from either of the origins. We use exponential functions to model such a dichotomy and the quick transition between small and large SV lengths. Given their distinct origins, we remove false small and large SV calls by requiring $n_{\mathrm{sr}}$ and $c_{\text {max }}$ to meet the following two conditions simultaneously:

$$
\begin{aligned}
& n_{\mathrm{sr}} \geq \operatorname{Round}\left[\left(t_{\mathrm{n}}-n_{\min }\right) \cdot e^{-\lambda(l-1)}+n_{\min }\right] \\
& c_{\max }>t_{\mathrm{c}}\left(1-e^{-\lambda(l-1)}\right)
\end{aligned}
$$

in which $t_{\mathrm{n}}$ is the threshold on the number of supportive reads for 1-bp SVs, $t_{\mathrm{c}}$ the threshold on the maximum centeredness for large SVs, $l$ the length of the SV in base pair, $n_{\text {min }}$ the minimum number of supportive reads for every SV identification (effectively the threshold on the number of supportive reads for large SVs), and $\lambda$ the exponential decay parameter that controls how fast the threshold changes between 1-bp and large SVs (Figure 7). $n_{\min }=2$ and $\lambda=1$ are used in all of our split-read analyses.

The error characteristics of different sequencing platforms are approximated by different error models. The

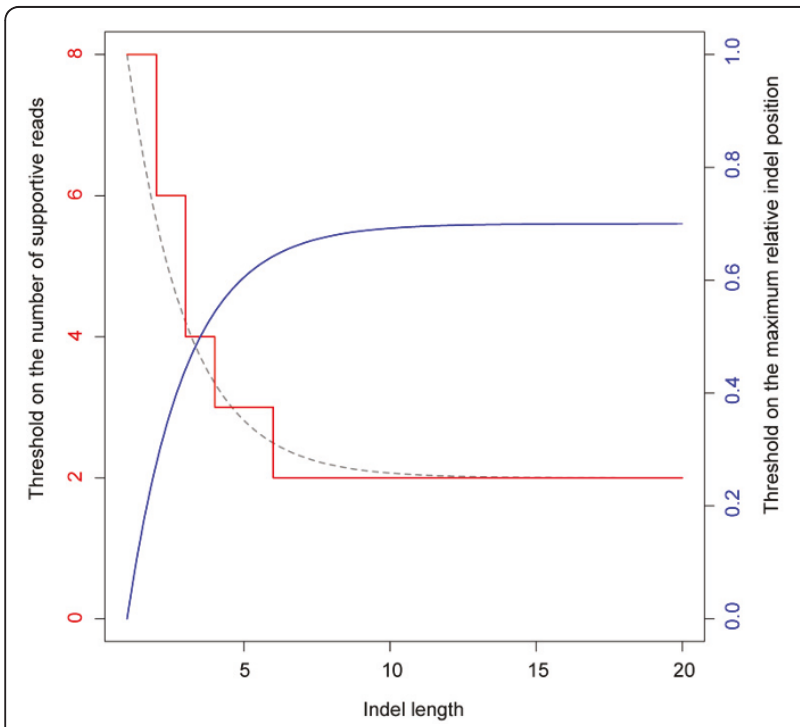

Figure $\mathbf{7}$ The curves of the threshold functions. Each SV call is scored by the number of supportive reads and the maximum centeredness in those reads. The thresholds on these two quantities are determined by two threshold functions, plotted as the read and the blue curves, respectively. The gray dashed curve is the threshold function for the number of supportive reads before rounding. The parameter values used for the shown functional curves are $\lambda=1, t_{n}$ $=8$, and $t_{\mathrm{c}}=0.7$ simplest model, which considers only 1-bp SVs, specifies the probability, $p_{\mathrm{e}}$, of 1 -bp SVs due to sequencing errors. After the initial call filtering, we perform a significant test for each 1-bp SV, where the null hypothesis is that the SV probability is the same as the probability specified by the error model. Because $p_{\mathrm{e}}$ stays the same for all sequence reads that contain the same 1-bp SV, we use the binomial distribution to calculate the $P$ value, which is the probability of seeing the same number and more of the reads having this SV out of the total number of reads covering this site, $n_{\mathrm{cr}}$, given the SV probability from the error model, $p_{\mathrm{e}}$ :

$$
P=\sum_{k=n_{\mathrm{sr}}}^{n_{\mathrm{cr}}} \frac{n_{\mathrm{cr}} !}{k !\left(n_{\mathrm{cr}}-k\right) !} p_{\mathrm{e}}^{k}\left(1-p_{\mathrm{e}}\right)^{n_{\mathrm{cr}}-k}
$$

After the Bonferroni correction for multiple tests, the null hypothesis is rejected if $P<0.01$.

Because of the increased likelihood of both under- and over-calling bases in homopolymers by 454 sequencing technology, for each SV that is a part of a homopolymer we perform a significant test after the initial call filtering, where the null hypothesis is that the SV probability is the same as the probability specified by the error model (Additional file 1, Figure S2). The calculation of the $P$-value is described above.

\section{Calibration of the number of SVs in a genomic region}

Previous steps will produce a set of SV calls for the assayed genomic region. Because the performance of our SR method can be assessed and quantified with the positive predictive value and the sensitivity by extensive simulation, we can use these error rates to derive less biased estimate of the number of SVs in that genomic region.

Given the number of SVs identified in sequence reads covering a genomic region (e.g., a chromosome or indeed the whole genome) to a certain depth, the total number of SVs of a certain length can be estimated using the positive predictive value and the sensitivity determined in a simulation data set with the same sequencing coverage:

$$
N_{l, c}^{\mathrm{est}}=\frac{P P V_{l, c}}{S_{l, c}} \cdot N_{l, c}^{\mathrm{obs}}
$$

in which $N_{l, c}^{\mathrm{obs}}, P P V_{l, c}$, and $S_{l, c}$ are the number of SVs, the positive predictive value, and the sensitivity for SVs of length $l$ (bp) observed in reads giving $c$-x sequence coverage. This method is not applicable to SVs of a certain length that are not observed (i.e., $N_{l, c}^{\text {obs }}=0$ ). For large SVs, it is more sensible to use a range of length, instead of discrete lengths. 


\section{Additional material}

Additional file 1: Supplementary materials. PDF file includes additional Methods and associated references, Tables S1 and S2, and Figures S1, S2, and S3.

\section{Acknowledgements}

This work was supported by an NIH grant (4R00LM009770-03) from the National Library of Medicine to ZDZ. We thank the 1000 Genomes Project for providing the sequence data and carrying out array-CGH validation.

\section{Author details}

'Department of Genetics, Albert Einstein College of Medicine, Bronx, NY 10461, USA. ${ }^{2}$ Department of Computer Science, Yale University, New Haven, CT 06520, USA. ${ }^{3}$ Interdepartmental Program in Computational Biology and Bioinformatics, Yale University, New Haven, CT 06520, USA. ${ }^{4}$ Department of Psychiatry and Behavioral Sciences, Stanford University, Stanford, CA 94305, USA. ${ }^{5}$ Department of Genetics, Stanford University, Stanford, CA 94305, USA

\section{Authors' contributions}

ZDZ implemented the method, performed the analyses, and drafted the manuscript. JD, HL, and AA helped with the implementation of the method. AEU and MS participated in the array-CGH validation for the 1000 Genomes Project. MG conceived of the study and helped to draft the manuscript. All authors read and approved the final manuscript.

Received: 26 January 2011 Accepted: 25 July 2011

Published: 25 July 2011

\section{References}

1. Campbell PJ, Stephens PJ, Pleasance ED, O'Meara S, Li H, Santarius T, Stebbings LA, Leroy C, Edkins S, Hardy C, et al: Identification of somatically acquired rearrangements in cancer using genome-wide massively parallel paired-end sequencing. Nature genetics 2008, 40(6):722-729.

2. Stenson PD, Mort M, Ball EV, Howells K, Phillips AD, Thomas NS, Cooper DN The Human Gene Mutation Database: 2008 update. Genome Med 2009, 1(1):13.

3. lafrate AJ, Feuk L, Rivera MN, Listewnik ML, Donahoe PK, Qi Y, Scherer SW, Lee C: Detection of large-scale variation in the human genome. Nat Genet 2004, 36(9):949-951.

4. Korbel JO, Urban AE, Affourtit JP, Godwin B, Grubert F, Simons JF, Kim PM, Palejev D, Carriero NJ, Du L, et al: Paired-end mapping reveals extensive structural variation in the human genome. Science 2007, 318(5849):420-426

5. Redon $R$, Ishikawa S, Fitch KR, Feuk L, Perry GH, Andrews TD, Fiegler $H$, Shapero MH, Carson AR, Chen W, et al: Global variation in copy number in the human genome. Nature 2006, 444(7118):444-454.

6. Sebat J, Lakshmi B, Troge J, Alexander J, Young J, Lundin P, Maner S, Massa $H$, Walker M, Chi M, et al: Large-scale copy number polymorphism in the human genome. Science 2004, 305(5683):525-528.

7. Tuzun E, Sharp AJ, Bailey JA, Kaul R, Morrison VA, Pertz LM, Haugen E, Hayden $\mathrm{H}$, Albertson D, Pinkel $\mathrm{D}$, et al: Fine-scale structural variation of the human genome. Nat Genet 2005, 37(7):727-732.

8. Perry GH, Ben-Dor A, Tsalenko A, Sampas N, Rodriguez-Revenga L, Tran CW Scheffer A, Steinfeld I, Tsang P, Yamada NA, et al: The fine-scale and complex architecture of human copy-number variation. Am J Hum Genet 2008, 82(3):685-695.

9. Pinkel D, Segraves R, Sudar D, Clark S, Poole I, Kowbel D, Collins C, Kuo WL, Chen $C$, Zhai $Y$, et al: High resolution analysis of DNA copy number variation using comparative genomic hybridization to microarrays. Nat Genet 1998, 20(2):207-211.

10. Selzer RR, Richmond TA, Pofahl NJ, Green RD, Eis PS, Nair P, Brothman AR, Stallings RL: Analysis of chromosome breakpoints in neuroblastoma at sub-kilobase resolution using fine-tiling oligonucleotide array CGH. Genes Chromosomes Cancer 2005, 44(3):305-319.

11. Urban AE, Korbel JO, Selzer R, Richmond T, Hacker A, Popescu GV, Cubells JF, Green R, Emanuel BS, Gerstein MB, et al: High-resolution mapping of DNA copy alterations in human chromosome 22 using high-density tiling oligonucleotide arrays. Proc Natl Acad Sci USA 2006, 103(12):4534-4539.

12. Abyzov A, Urban AE, Snyder M, Gerstein M: CNVnator: An approach to discover, genotype and characterize typical and atypical CNVs from family and population genome sequencing. Genome research 2011.

13. Wheeler DA, Srinivasan M, Egholm M, Shen Y, Chen L, McGuire A, He W, Chen YJ, Makhijani V, Roth GT, et al: The complete genome of an individual by massively parallel DNA sequencing. Nature 2008, 452(7189):872-876

14. Xie C, Tammi MT: CNV-seq, a new method to detect copy number variation using high-throughput sequencing. BMC Bioinformatics 2009, $10: 80$

15. Zhang ZD, Gerstein MB: Detection of copy number variation from array intensity and sequencing read depth using a stepwise Bayesian model. BMC Bioinformatics 2010, 11:539.

16. Chen J, Kim YC, Jung YC, Xuan Z, Dworkin G, Zhang Y, Zhang MQ, Wang SM: Scanning the human genome at kilobase resolution. Genome Res 2008, 18(5):751-762.

17. Kidd JM, Cooper GM, Donahue WF, Hayden HS, Sampas N, Graves T, Hansen N, Teague B, Alkan C, Antonacci F, et al: Mapping and sequencing of structural variation from eight human genomes. Nature 2008 453(7191):56-64

18. Korbel JO, Abyzov A, Mu XJ, Carriero N, Cayting P, Zhang Z, Snyder M Gerstein MB: PEMer: a computational framework with simulation-based error models for inferring genomic structural variants from massive paired-end sequencing data. Genome Biol 2009, 10(2):R23.

19. Abyzov A, Gerstein M: AGE: defining breakpoints of genomic structural variants at single-nucleotide resolution, through optimal alignments with gap excision. Bioinformatics 2011, 27(5):595-603

20. Chen K, Wallis JW, McLellan MD, Larson DE, Kalicki JM, Pohl CS, McGrath SD, Wendl MC, Zhang Q, Locke DP, et al: BreakDancer: an algorithm for high-resolution mapping of genomic structural variation. Nat Methods 2009, 6(9):677-681.

21. Hormozdiari F, Alkan C, Eichler EE, Sahinalp SC: Combinatorial algorithms for structural variation detection in high-throughput sequenced genomes. Genome Res 2009, 19(7):1270-1278.

22. Lee S, Hormozdiari F, Alkan C, Brudno M: MoDIL: detecting small indels from clone-end sequencing with mixtures of distributions. Nat Methods 2009, 6(7):473-474

23. Medvedev $P$, Stanciu M, Brudno M: Computational methods for discovering structural variation with next-generation sequencing. Nature methods 2009, 6(11 Suppl):S13-20

24. Mills RE, Walter K, Stewart C, Handsaker RE, Chen K, Alkan C, Abyzov A, Yoon SC, Ye K, Cheetham RK, et al: Mapping copy number variation by population-scale genome sequencing. Nature 2011, 470(7332):59-65.

25. Ye K, Schulz MH, Long Q, Apweiler R, Ning Z: Pindel: a pattern growth approach to detect break points of large deletions and medium sized insertions from paired-end short reads. Bioinformatics 2009, 25(21):2865-2871.

26. Levy S, Sutton G, Ng PC, Feuk L, Halpern AL, Walenz BP, Axelrod N, Huang J, Kirkness EF, Denisov G, et al: The diploid genome sequence of an individual human. PLOS Biol 2007, 5(10):e254.

27. Kent WJ: BLAT-the BLAST-like alignment tool. Genome Res 2002 12(4):656-664.

28. Li H, Durbin R: Fast and accurate long-read alignment with BurrowsWheeler transform. Bioinformatics 2010, 26(5):589-595.

29. Langmead B, Trapnell C, Pop M, Salzberg SL: Ultrafast and memoryefficient alignment of short DNA sequences to the human genome. Genome Biol 2009, 10(3):R25.

doi:10.1186/1471-2164-12-375

Cite this article as: Zhang et al:: Identification of genomic indels and structural variations using split reads. BMC Genomics 2011 12:375. 